\title{
Microfluidics approach to investigate foam hysteretic behaviour
}

\author{
Leslie Labarre $^{1} \cdot$ Daniele Vigolo $^{1}$ (1)
}

Received: 29 May 2019 / Accepted: 24 October 2019 / Published online: 8 November 2019

(c) The Author(s) 2019

\begin{abstract}
Foam stability often refers to the foam left to evolve with time in static conditions. However, in everyday life, foams are submitted to numerous deformations. A feature of foam stability is represented by the foam's ability to resist to the deformation and to recover its initial properties after deformation. The technique developed here allows for a qualitative evaluation of the property of foam recovery after a deformation in a flow-focusing microfluidic device. The foam hysteretic behaviour was evaluated by introducing the analogous of a standard three-step test in which the recovery of viscosity is commonly studied over three deformation stages. The foam behaviour is analysed over an induced cycle of ascendant and descendant deformation at the wall, well controlled by varying the gas pressure for a constant liquid pressure. Thus, the recovery of the two-row foam pattern used as reference is studied after a high deformation phase corresponding to the bamboo pattern and the level of hysteresis is measured qualitatively. The samples investigated comprise a range of Newtonian aqueous solutions containing $5 \mathrm{cmc}$ (critical micellar concentration) of sodium dodecyl sulphate (SDS). A retardation effect was observed leading to hysteresis caused by the increase in viscosity. A higher surface elasticity produced a smaller but non-negligible hysteresis due to an excess in elastic energy caused by the increase of the duration of the bubble rearrangements. The present study has gone some way towards enhancing our understanding of the mechanisms triggering or enhancing foam hysteresis in a microchannel. The findings will be of interest to many industrial processes where foams are submitted to a series of deformation steps along the process line from food industrial applications to biological systems.
\end{abstract}

\section{Graphical abstract}

A schematic of the three-step test consisting in an ascending and descending pressure ramps obtained by varying the gas pressure for a constant liquid pressure.

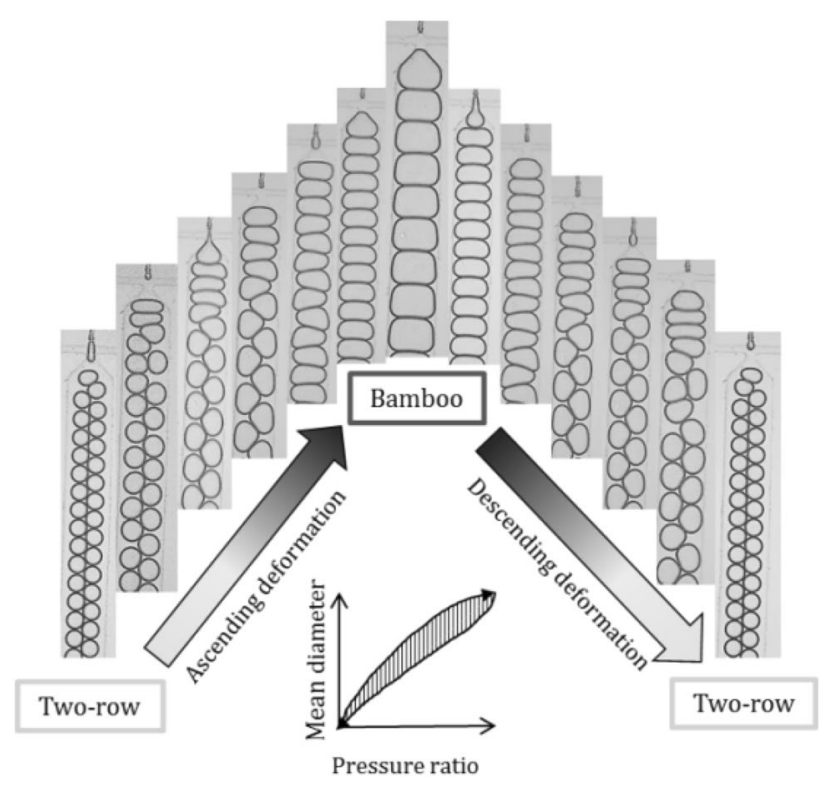

Keywords Foam $\cdot$ Hysteresis $\cdot$ Retardation $\cdot$ Microfluidics

Extended author information available on the last page of the article 


\section{Introduction}

Aqueous foams are dispersions of gas bubbles in a liquid continuous phase. They are used in a wide range of applications from enhanced oil recovery (EOR) (Quennouz et al. 2014; Guo and Aryana 2016; Yekeen et al. 2018) to food industry (Skurtys, Bouchon and Aguilera 2008; Laporte et al. 2016) and biological applications such as biocompatible scaffolds (Chung et al. 2009; Costantini et al. 2015; Andrieux et al. 2017).

Aqueous foams can behave like a shear-thinning fluid over time after being subjected to deformation (Bekkour and Scrivener 1998). This property, called "thixotropy", plays an important role in foam stability (Mewis 1979; de Souza Mendes 2009; Mewis and Wagner 2009; Larson 2015). Thixotropy implies the creation of a change in foam properties before and after the deformation, thus, hysteresis.

Foam rheology and more precisely foam thixotropy or hysteretic behaviour is mainly studied by rheometry (Miquelim and Da Silva Lannes 2009). The two most common tests employed to study how a fluid recovers after deformation are the three-step test and the creep test (Mezger 2014). The three-step test consists of three consecutive stages of rest, deformation and rest. The initial rest viscosity is taken as reference for the study of the fluid recovery after a gradual or sharp deformation. This test has been adapted to fragile products such as foams by applying a non-destructive deformation to preserve the sample (Asnacios et al. 1999). An example of a thixotropy study by performing an oscillatory test within the linear viscoelastic range is given in Marze et al. (2009).

However, foams evolve over time due to the simultaneous destabilising events which are the key actors of its stability kinetic (e.g., drainage, coalescence and coarsening) (SaintJalmes et al. 2004; Pitois et al. 2005; Saint-Jalmes 2006). This ageing process, in addition to the quantity of product required and the cost of the equipment, demonstrates the limitations of macroscale rheology to study fragile and complex products such as foams.

A decade ago, a novel and innovative use of microfluidics was developed at first to measure the viscosity of single phase fluids (Guillot et al. 2006) and further to measure the properties of viscoelastic fluids such as the relaxation times (Pipe and McKinley 2009; Galindo-Rosales et al. 2013; Koser et al. 2013). Microfluidics rheometry matches the standards of its macroscopic counterpart with the advantages of requiring much less amount of sample and lower cost. Thus, extending this use of microfluidics to two-phase fluids can bring a new perspective in studying how the shear viscosity, the surface viscosity and other properties influence the rheological behaviour of the foam. Here, a new way to study and to evaluate the parameters influencing the property of recovery of foam after a gradual deformation at the wall was implemented. These parameters comprise the range of properties affecting the microstructure of the foam from the continuous phase viscosity, the surface tension and the interfacial properties such as the surface elasticity.

Interestingly, a microstructural hysteresis was observed in an expansion-contraction microchannel (Drenckhan et al. 2005). Researchers highlighted the link between the microstructural hysteresis occurring in the microchannel while the foam was continuously flowing and the foam liquid fraction. The importance of the surface drag in the development of the hysteresis was pointed out. In the present work, the scope is to study the parameters influencing the induced microstructural hysteresis during the deformation cycle in a fixed geometry. The influence of the surface drag is characterised by controlling the level of deformation at the wall via the fine-tuning of the gas and liquid inlet pressures.

\section{Experimental}

\subsection{Materials}

SDS is a common anionic surfactant used in a wide range of foam industrial applications. A concentration above $\mathrm{cmc}$ (critical micellar concentration) is chosen to ensure a constant concentration of surface-active agent throughout the whole experiment. Glycerol (GLY, Sigma-Aldrich) is used at 20 and $40 \%$ (wt.) to increase the dynamic viscosity of the reference solution. Dodecanol (DOH, Sigma-Aldrich) is added to the reference solution at a concentration of $0.15 \mathrm{~g}$ $\mathrm{L}^{-1}$ to study the effect of surface elasticity on the foam hysteretic behaviour. The surface tensions of the different solutions investigated are measured via maximum bubble pressure technique (SINTERFACE, BPA-1) and summarized in Table 1.

The viscosity of each solution was investigated via a shear rate sweep from 5 to $100 \mathrm{~s}^{-1}$ via a cone-plate geometry (2-degree truncation, $60 \mathrm{~mm}$ diameter) on a rheometer (DHR-1, TA) (Fig. 1).

All the solutions show a Newtonian behaviour with a steady viscosity ranging from $0.6 \mathrm{mPa}$ s for the reference

Table 1 Summary of foaming solutions investigated: (A) $5 \mathrm{cmc}$ SDS, (B) $5 \mathrm{cmc}$ SDS $+20 \%$ (wt.) glycerol, (C) $5 \mathrm{cmc}$ SDS $+40 \%$ (wt.) glycerol and (D) $5 \mathrm{cmc} \mathrm{SDS}+0.15 \mathrm{~g} \mathrm{~L}^{-1} \mathrm{DOH}$

\begin{tabular}{ll}
\hline Solutions & $\begin{array}{l}\sigma \text {, surface ten- } \\
\text { sion }\left(\mathrm{mN} \mathrm{m}^{-1}\right)\end{array}$ \\
\hline (A) $5 \mathrm{cmc} \mathrm{SDS}$ & 36.3 \\
(B) $5 \mathrm{cmc} \mathrm{SDS}+20 \% \mathrm{wt} \mathrm{GLY}$ & 37.6 \\
(C) $5 \mathrm{cmc} \mathrm{SDS}+40 \% \mathrm{wt} \mathrm{GLY}$ & 45.5 \\
(D) $5 \mathrm{cmc} \mathrm{SDS}+0.15 \mathrm{~g} \mathrm{~L}^{-1} \mathrm{DOH}$ & 36.7
\end{tabular}


solution (A), and reference + DOH (D), $1.5 \mathrm{mPa}$ s for the reference $+20 \%$ (wt.) glycerol (B) to $3.5 \mathrm{mPa}$ s for the reference $+40 \%$ (wt.) glycerol (C).

\subsection{Experimental setup}

The microfluidic device used consists of a rectangular crosssection microchannel of $25 \mu \mathrm{m}$ (d) depth with a $50 \mu \mathrm{m}\left(\mathrm{w}_{1}\right)$ wide gas inlet which meets at a flow-focusing junction with

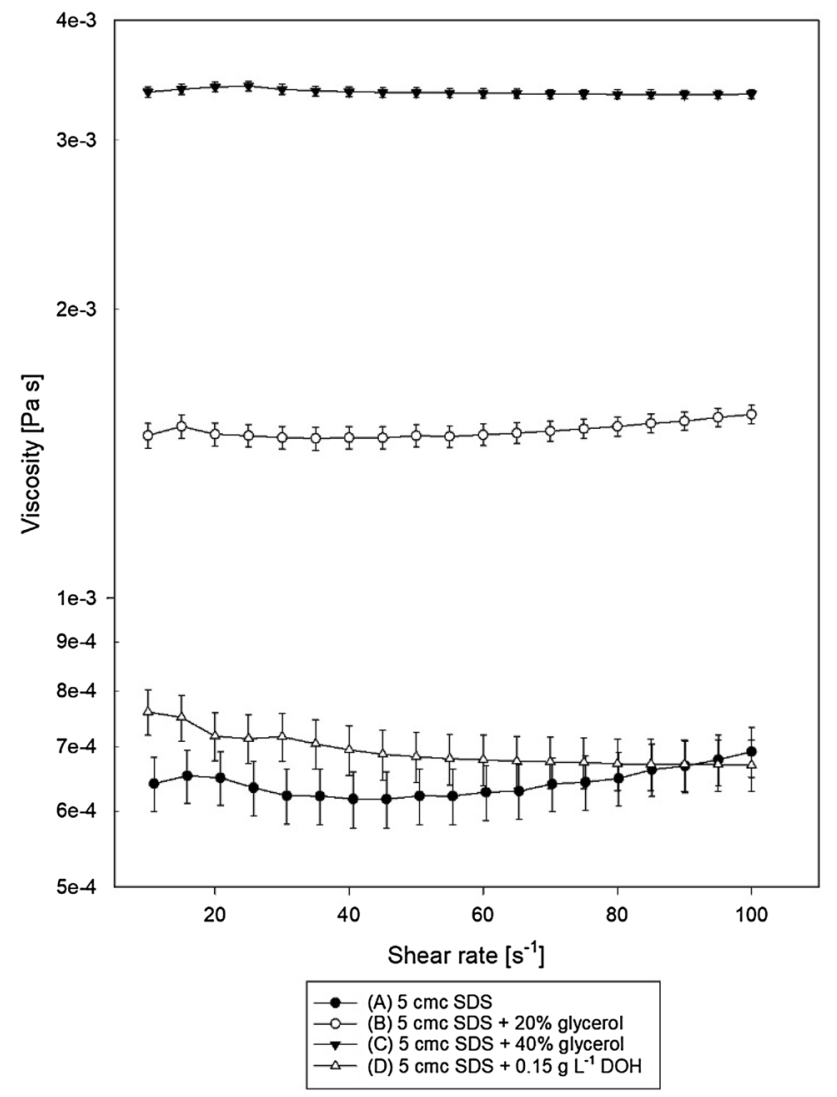

Fig. 1 Rheological properties of solutions investigated: (A) $5 \mathrm{cmc}$ SDS, (B) $5 \mathrm{cmc}$ SDS $+20 \%$ (wt.) glycerol, (C) $5 \mathrm{cmc}$ SDS $+40 \%$ (wt.) glycerol and (D) $5 \mathrm{cmc} \mathrm{SDS}+0.15 \mathrm{~g} \mathrm{~L}^{-1} \mathrm{DOH}$ two $100 \mu \mathrm{m}\left(\mathrm{w}_{2}\right)$ wide liquid inlets as shown in Fig. 2. The foam is formed and studied in a $280 \mu \mathrm{m}\left(\mathrm{w}_{3}\right)$ wide channel. Two reservoirs of air and liquid are connected via a pressure controller (OB1 MK3, Elveflow) to the two inlets via $0.020^{\prime \prime} \times 0.060 "$ OD Tygon microbore tubing (ColeParmer Instrument Co. Ltd., UK) to accurately control the gas and liquid inlet pressures. The device is fabricated in polydimethylsiloxane (PDMS, SYLGARD ${ }^{\text {TM }} 184$ Silicone Elastomer Kit, Dow Chemical) via soft lithography (Kim et al. 2008). The PDMS device is then irreversibly bonded to a microscope glass slide by corona discharge (Mark et al. 2008). To assure a homogeneous foam formation in the channel (Cubaud et al. 2006), the device is surface treated to become hydrophilic via a layer-by-layer technique by flowing alternately segments of poly(allylamine hydrochloride) (PAH, Sigma-Aldrich) and poly(sodium 4-styrenesulfonate) (PSS, Sigma-Aldrich) solutions (both $0.1 \% \mathrm{w} / \mathrm{v}$ in $0.5 \mathrm{M}$ aqueous $\mathrm{NaCl}$ solution) with aqueous $\mathrm{NaCl}$ washing solution $(0.1 \mathrm{M})$ segments in between as described in Bauer et al. (2010).

\subsection{Methods}

A foam regime map (FRM), which gives a detailed view of all the different foam patterns available for a specific geometry, is first generated for each solution by changing the gas and liquid inlet pressures between 200 and 1400 mbar. Thus, areas of steady patterns of bamboo and two-row foam can be identified and selected for the study. The "bamboo" pattern or "hex-one" (Garstecki and Whitesides 2006) is described as a single layer of bubbles containing only one bubble in the full width of the channel. The "two-row" pattern or "hextwo" (Garstecki and Whitesides 2006) can be depicted as a single layer containing two rows of bubbles in the width of the channel. In the bamboo pattern, the deformation at the wall during the continuous foam generation and flow in the channel is considered as higher than the two-row pattern due to the largest surface area in contact with the wall. Indeed, the average apparent bubble superficial area at the wall was estimated by image analysis. It was found that the bamboo
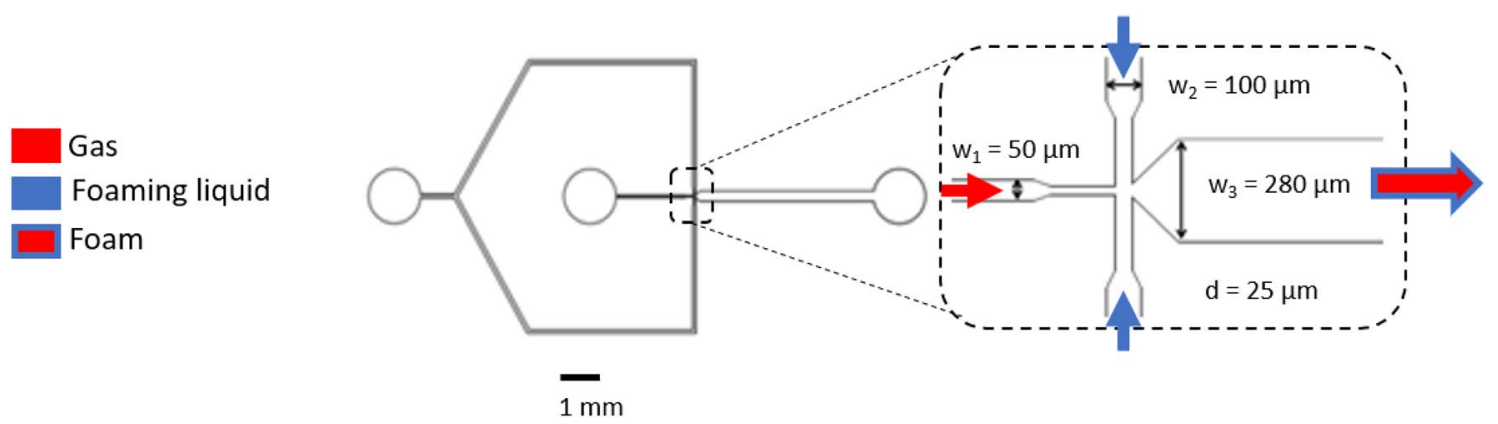

Fig. 2 Schematic of the $25 \mu \mathrm{m}$ depth flow-focusing device (left) and a zoomed-in view of the flow-focusing junction (right) 
pattern superficial area varied from 30,000 to $40,000 \mu \mathrm{m}^{2}$, whereas the two-row pattern presented an average surface ranging from 4000 to $6000 \mu^{2}$.

Two ways can be employed to vary the degree of deformation at the wall: by changing the pressure sets (gas and liquid inlet pressures) which will also modify the gas and liquid flow rates and the foam pattern, or by altering the pressure ramp (i.e., the variation of pressure over time). Indeed, the amount of energy needed to deform the foam varies significantly with the pressure ramp. By modifying the pressure ramp from 0.5 to $2 \mathrm{mbar} \mathrm{s}^{-1}$, the foam lamella has less time to rearrange/recover for the same level of deformation applied and so to reach its equilibrium state by dissipating the energy to the surrounding. The pressure sets are chosen so that the beginning and the end of the deformation cycle present a steady foam pattern.

It was shown (Raven and Marmottant 2006, 2009) that for a steady liquid flow rate, increasing the gas pressure will augment the gas flow rate up to a plateau due to a self-regulation mechanism for which the foam structure changes from bamboo to two-row continuously. In the present work, the foam velocity during the deformation is mainly controlled by the liquid pressure.

The evolution of the apparent mean bubble diameter during one deformation cycle is obtained via image analysis of snap shots collected by a high-speed camera (Photron SA5) at up to 20,000 frames per second (fps) via an inverted optical microscope (Nikon $\mathrm{Ti}-\mathrm{U}$ ) for specific pressure ratio $\left(r=\frac{P_{\text {gas }}}{P_{\text {liq }}}\right)$ in the ascending and descending pressure ramps.

From each snap shot, the apparent mean bubble diameter is obtained by calculating the equivalent diameter of a circle having the same area of the one obtained for the bubble using the ImageJ software (Schneider et al. 2012), and normalised by the channel width $(280 \mu \mathrm{m})$. By plotting the normalised apparent mean bubble diameter versus the pressure ratio, the hysteresis is then evaluated by measuring the area between the ascending and descending curves.

The $\mathrm{T} 1$ rearrangement durations are estimated via image analysis using the ImageJ software (Schneider et al. 2012).

\section{Results and discussions}

The foam regime map illustrating the reference solution and the other foaming liquids are introduced in Fig. 3.

The impact of the viscosity and surface elasticity on the foam regime map (FRM) is already noticeable.

First, the effect of viscosity for a fixed surface tension is analysed by comparing the FRM in Fig. $3 b$ to the reference FRM in Fig. 3a. It is found that the increase in viscosity of the foaming solution generates smaller bubble diameters for the two-row pattern. Indeed, at 400 mbar gas and
600 mbar liquid pressures, the average bubble area obtained decreases from $12,891 \mu^{2}$ for the $5 \mathrm{cmc}$ SDS solution down to $5530 \mu^{2}$ for the $20 \%$ glycerol solution. At $40 \%$ glycerol, a two-row pattern depicting a mean bubble area of $6620 \mu \mathrm{m}^{2}$ is formed. Despite the increase in viscosity, which tends to create smaller bubbles for a given flow rate, the $40 \%$ glycerol solution shows an increase in surface tension from 36.7 to $45.5 \mathrm{mN} \mathrm{m}^{-1}$ at $25{ }^{\circ} \mathrm{C}$ responsible for the creation of slightly larger bubbles compared to the sample containing $20 \%$ glycerol.

From Ruiz et al. (2008) describing the evolution of the micellization of SDS with the glycerol concentration in solution, it was demonstrated that the addition of glycerol increases the solubility of the SDS hydrophobic part. As a result, the miscibility of the hydrophilic part of the molecule decreases as the solution presents a higher affinity to the hydrophobic component. Thus, the adsorption of SDS molecules at the interface is limited and the surface tension increases.

Then, the increase in surface elasticity results in the extension of the bamboo pattern towards larger gas pressures in FRM (D). The interface submitted to surface drag at the wall is "stiffer" albeit having the same surface tension as the reference solution. From Karakashev and Nguyen (2007), DOH molecules adsorb at the gas-liquid interface and limit the surface mobility of surfactant molecules due to their carbon backbone.

From this preliminary characterisation step, two steady foam patterns from the FRM are identified as low deformation (two-row pattern) and high deformation (bamboo pattern) at the wall. The response of the foam to the deformation is identified via the comparison of the evolution of the mean bubble diameter over time in an ascending versus a descending ramp.

\subsection{Reference solution: $5 \mathrm{cmc}$ SDS}

The evolution of the hysteresis with the pressure ramps of 0.5 and 2 mbar s$^{-1}$ is evaluated in this section for low (P1, corresponding to $600 \mathrm{mbar}$ liquid inlet pressure for a gas pressure ranging from 400 to $600 \mathrm{mbar}$ ) and high (P2, corresponding to $800 \mathrm{mbar}$ liquid inlet pressure for a gas pressure ranging from 500 to $800 \mathrm{mbar}$ ) pressure sets. Figure 4 shows the evolution of the hysteresis versus the pressure ratio per pressure ramp and set. Each hysteresis value is obtained from the average of two experiments with three repeats for each setting (ramp and set). From the inset, the foam formed from the $5 \mathrm{cmc}$ SDS solution does not present any hysteresis both at the low- and highpressure ramps and sets.

The average of the areas in between the ascending and descending curves obtained for several experiments is 

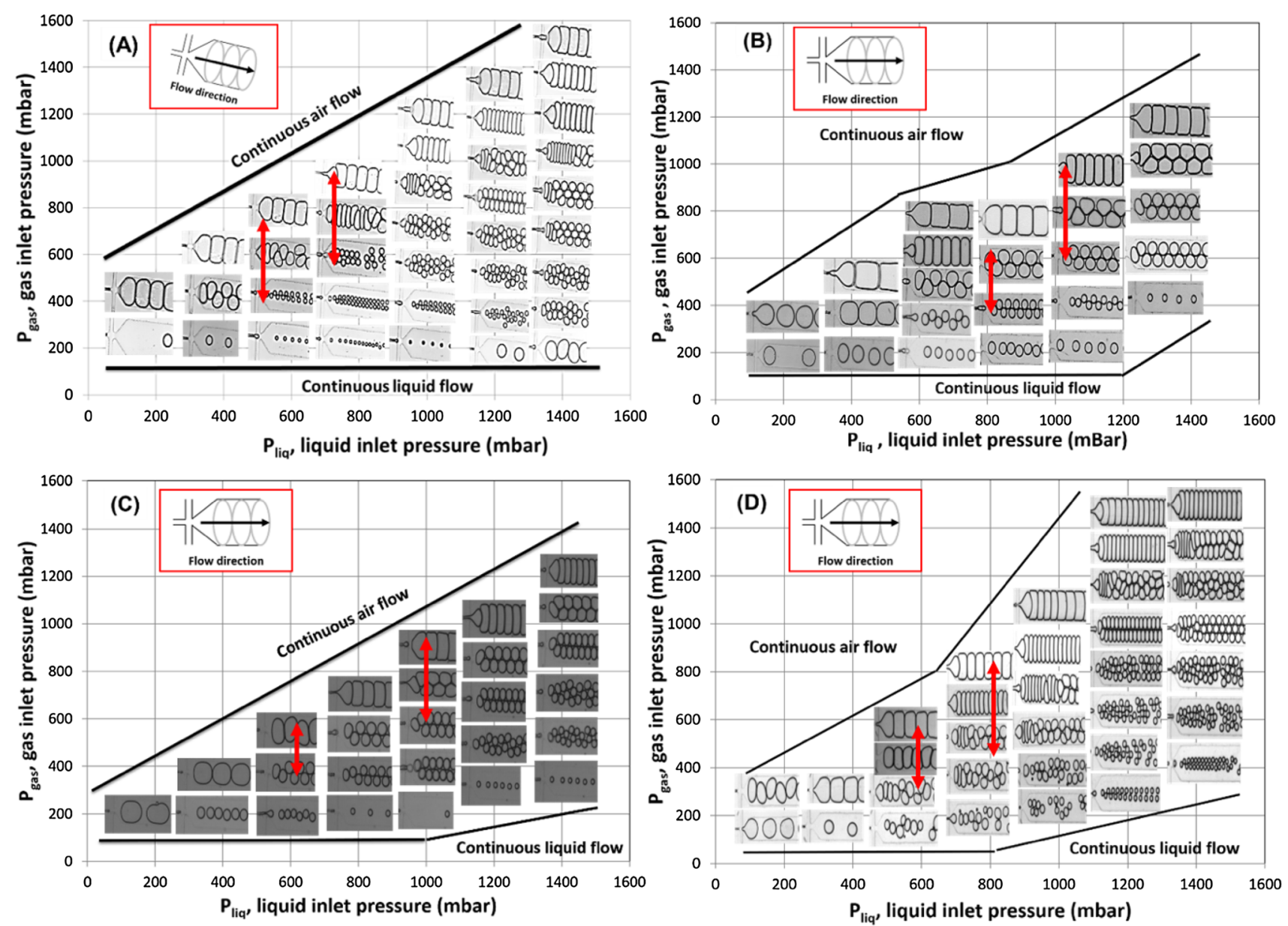

Fig. 3 Foam regime maps plotting the gas inlet pressure $\left(P_{\text {gas }}\right)$ versus the liquid inlet pressure $\left(P_{\text {liq }}\right)$ for the following solutions: a $5 \mathrm{cmc}$ SDS (reference system), b $5 \mathrm{cmc}$ SDS $+20 \%$ (wt.) glycerol, c $5 \mathrm{cmc}$

SDS $+40 \%$ (wt.) glycerol, and $\mathbf{d} 5 \mathrm{cmc}$ SDS $+0.15 \mathrm{~g} \mathrm{~L}^{-1} \mathrm{DOH}$. The red arrows from left to right represent the low (P1) and high (P2) pressure ranges investigated for the formulation

taken as the "noise" of the measurement which represents our zero reference value to determine if a foam presents hysteretic behaviour or not. Thus, the value obtained for this sample, $1.5 \times 10^{-3} \pm 6.5 \times 10^{-4}$ (represented by the red continuous line in Figs. 4, 7 and 9) is considered as the qualitative reference number describing negligible hysteresis.

\subsection{Effect of viscosity}

Two different cases are presented to highlight the effect of viscosity and the combined influence of viscosity and surface tension on foam hysteresis.

\subsection{1 $5 \mathrm{cmc}$ SDS +20 wt. \% glycerol}

The effect of the increase in pressure ramp from 0.5 to 2 mbar s$^{-1}$ is depicted in Fig. 5 for low- (P1, corresponding

to 800 mbar liquid inlet pressure for a gas pressure ranging from 500 to 800 mbar) and for high- (P2, corresponding to 1000 mbar liquid inlet pressure for a gas pressure ranging from 800 to 980 mbar) pressure sets.

From the inset in Fig. 5, hysteresis is significantly emphasized by the increase in viscosity for a fixed surface tension. Hysteresis increases with both pressure sets and ramps. However, a synergetic effect is observed at P2 and $2 \mathrm{mbar} \mathrm{s}^{-1}$. Indeed, hysteresis is doubled with the pressure ramp at $\mathrm{P} 1$ and is multiplied by almost 20 at $\mathrm{P} 2$.

\subsection{2 $5 \mathrm{cmc}$ SDS +40 wt. \% glycerol}

The increase in glycerol concentration impacts dramatically the foam recovery property throughout the deformation cycle. The impact of the increase in pressure ramp from 0.5 to 2 mbar s$^{-1}$ is shown in Fig. 6 for low- (P1, corresponding to 600 mbar liquid inlet pressure for a gas pressure ranging from 400 to $600 \mathrm{mbar})$ and in Fig. 7 for high- (P2, 


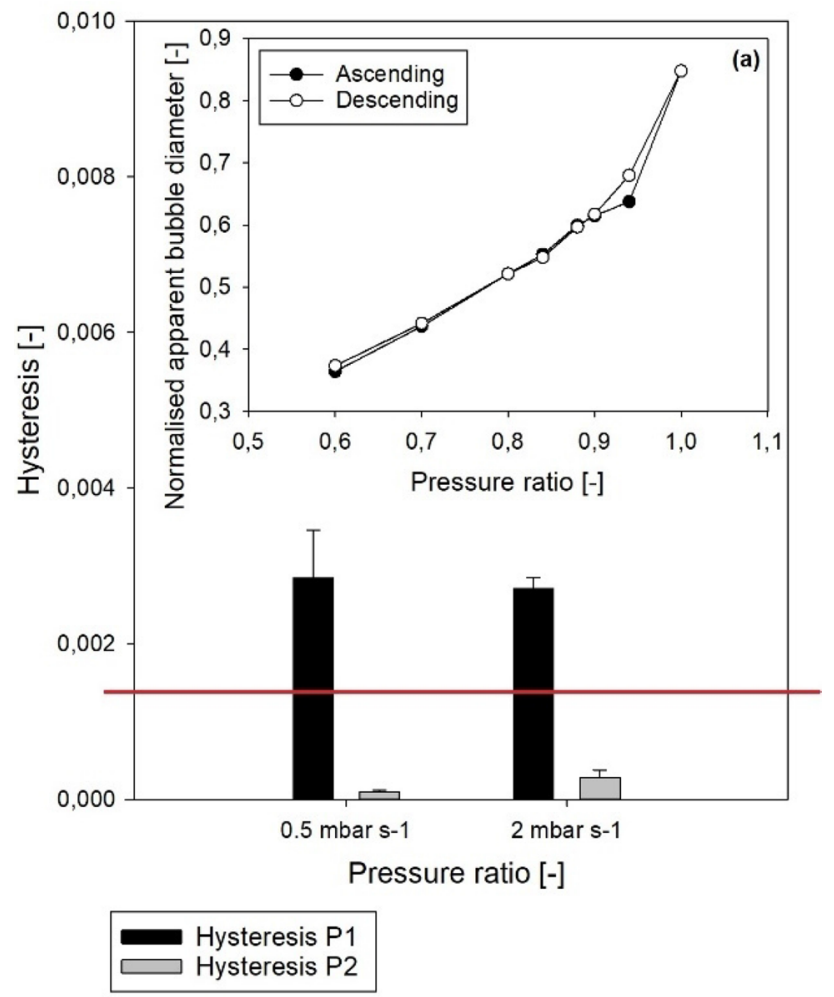

Fig. 4 Hysteresis evolution for $5 \mathrm{cmc}$ SDS at low- (P1, black) and high-pressure (P2, grey) sets for both pressure ramps (0.5 and $2 \mathrm{mbar} \mathrm{s}^{-1}$ ). In the inset, a typical curve for P1 at $0.5 \mathrm{mbar} \mathrm{s}^{-1}$

corresponding to 1000 mbar liquid inlet pressure for a gas pressure ranging from 600 to 900 mbar) pressure sets.

From Fig. 6, for a low-pressure set, a large hysteresis is observable. The resistance to the deformation at the wall is higher due to the increase in viscosity. Thus, once the bamboo/annular pattern has been reached, after the high deformation plateau, in the descending ramp, the foam "holds" for longer the bamboo pattern. Indeed, at higher viscosity, the foam takes longer time to rearrange itself. The foam response to the deformation is then slower. This can cause retardation in the foam response to the deformation leading to an enhanced hysteretic behaviour.

However, this hysteresis is reduced with the increase in pressure ramp at P1. Indeed, more energy per second is given to the foam to operate the change in pattern due to the increase in gas pressure. Despite the increase in viscosity which slows down the process, the retardation is limited as more energy is given to the foam to respond to the deformation. Thus, the foam recovery after the high deformation stage is improved.

Furthermore, coalescence (merging of two neighbour bubbles due to film thinning) was increased towards the end of the ascending ramp. In the channel, drainage and coarsening are prevented: gravity is negligible, and the
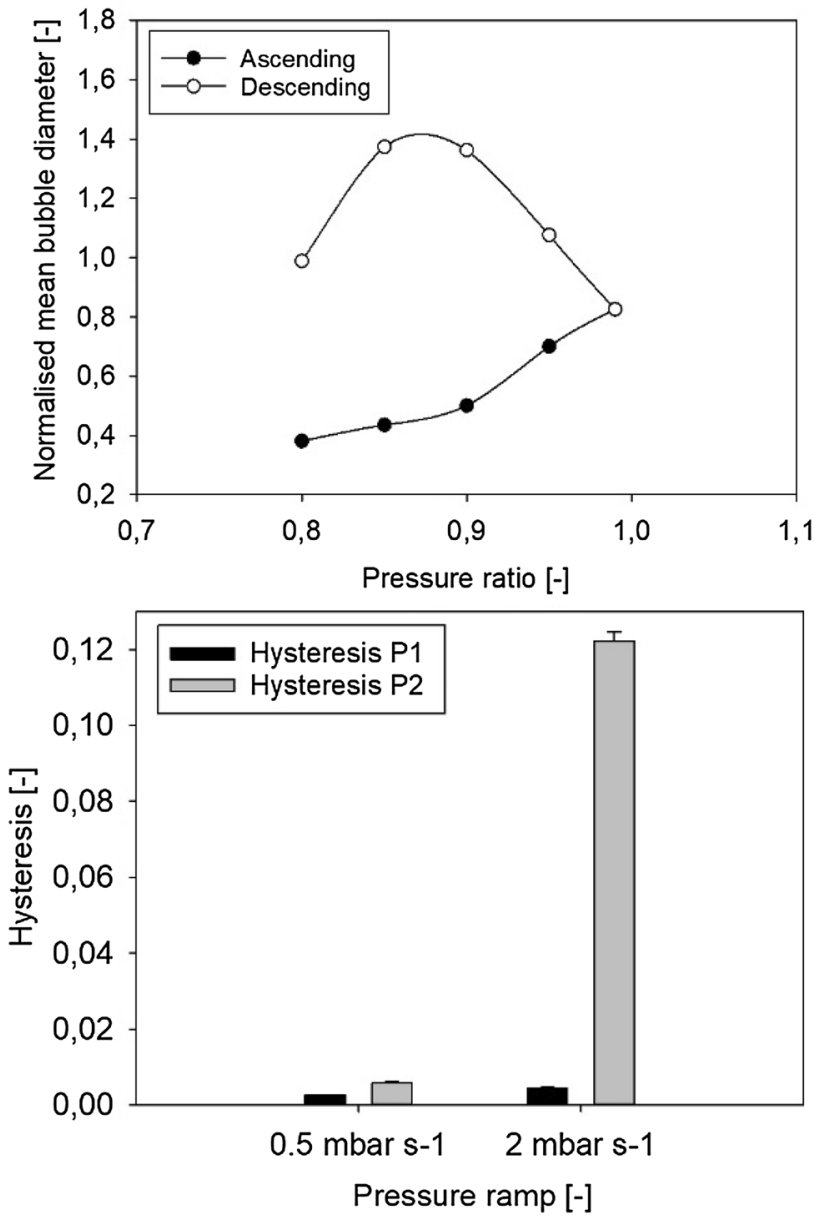

Fig. 5 Hysteresis evolution for $5 \mathrm{cmc}$ SDS $+20 \%$ (wt.) glycerol at low- (P1, black) and high (P2, grey)-pressure sets for both pressure ramps $\left(0.5\right.$ and $\left.2 \mathrm{mbar} \mathrm{s}^{-1}\right)$. In the inset, a typical curve for P2 at 2 mbar s $^{-1}$

bubble distribution is monodispersed. Thus, the hysteresis observed in the channel is mainly due to the factors (surface tension, viscosity) impacting the thinning of the film between two neighbouring bubbles, leading to film breakage and coalescence.

In the present study, the main parameter that can cause film breakage is the raise in surface tension driven by the addition of glycerol in solution. In this peculiar case, the hysteresis is primarily triggered by the change in viscosity but then enhanced by the surface tension increase.

From Fig. 7, at high-pressure set, the foam response to the deformation is different: the hysteresis is negligible at low-pressure ramp and rises up to 9 times the initial value with the change in pressure ramp.

Foams by definition are yield stress fluids (Höhler and Cohen-Addad 2005; Cohen-Addad and Höhler 2014). The yield stress is defined as the stress at which the foam behaviour changes from solid-like to liquid-like. Unlike 


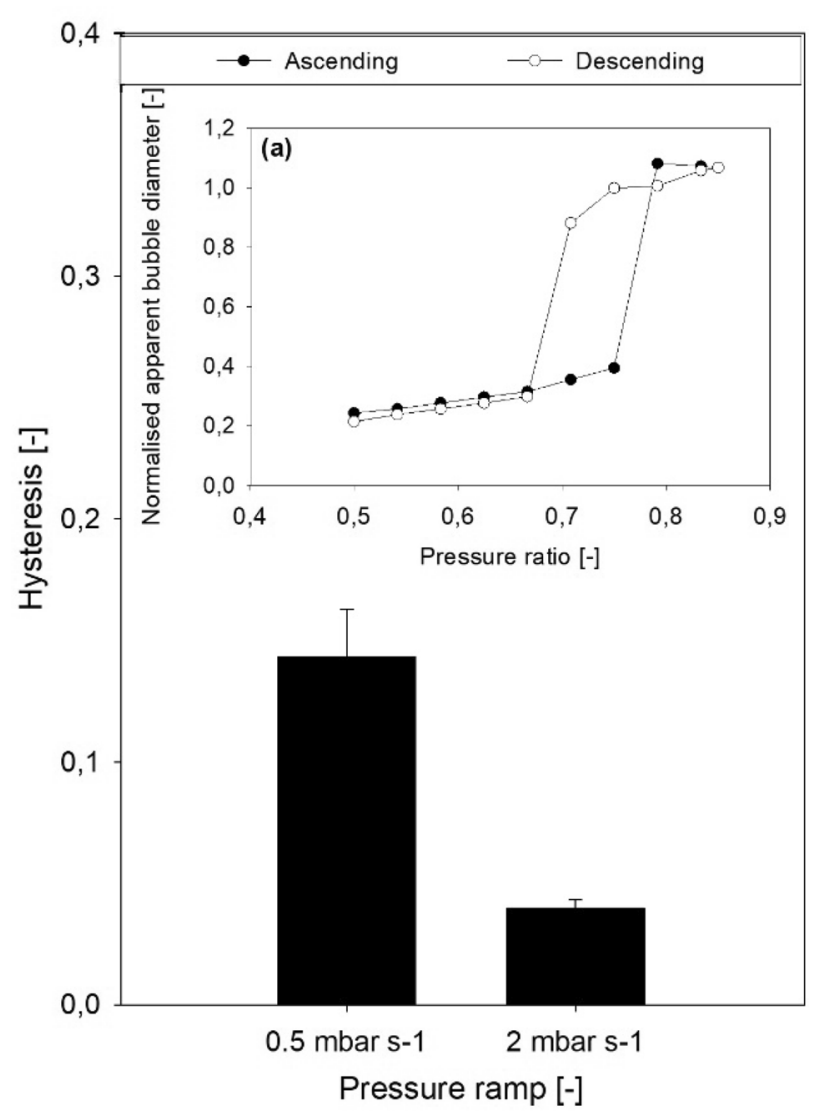

Hysteresis P1

Fig. 6 Hysteresis evolution for $5 \mathrm{cmc}$ SDS $+40 \%$ (wt.) glycerol at low-pressure set (P1, black) for both pressure ramps $(0.5$ and $\left.2 \mathrm{mbar} \mathrm{s}^{-1}\right)$. In the inset, a typical curve for P1 at $2 \mathrm{mbar} \mathrm{s}^{-1}$

the lower pressure case, here increasing the pressure ramp enhances the hysteretic behaviour.

This difference in foam response is due to the change in pressure set. Below the yield stress, foam will behave as a solid-like (elastic) fluid and above, as a liquid-like (viscous) fluid. This change in behaviour leads to different foam responses to the deformation. Therefore, it is considered that the foam generated presents a critical pressure set $\mathrm{P}_{\mathrm{c}}$ located between $\mathrm{P} 1$ and $\mathrm{P} 2$ at which the rheological behaviour changes from solid-like (elastic) to liquid-like (viscous).

Thereby, at $\mathrm{P} 2>P_{\mathrm{c}}$, the foam is characterised by a liquidlike behaviour. Giving more energy to the system per second will have a counter effect: the interface will be more subjected to the deformation, and hysteresis will increase with the ramp.

On the contrary, at $\mathrm{P} 1<P_{\mathrm{c}}$, the foam behaves as a solidlike fluid. In this case, providing more energy to the system per second reduces the hysteresis by helping the system to respond faster to the deformation.

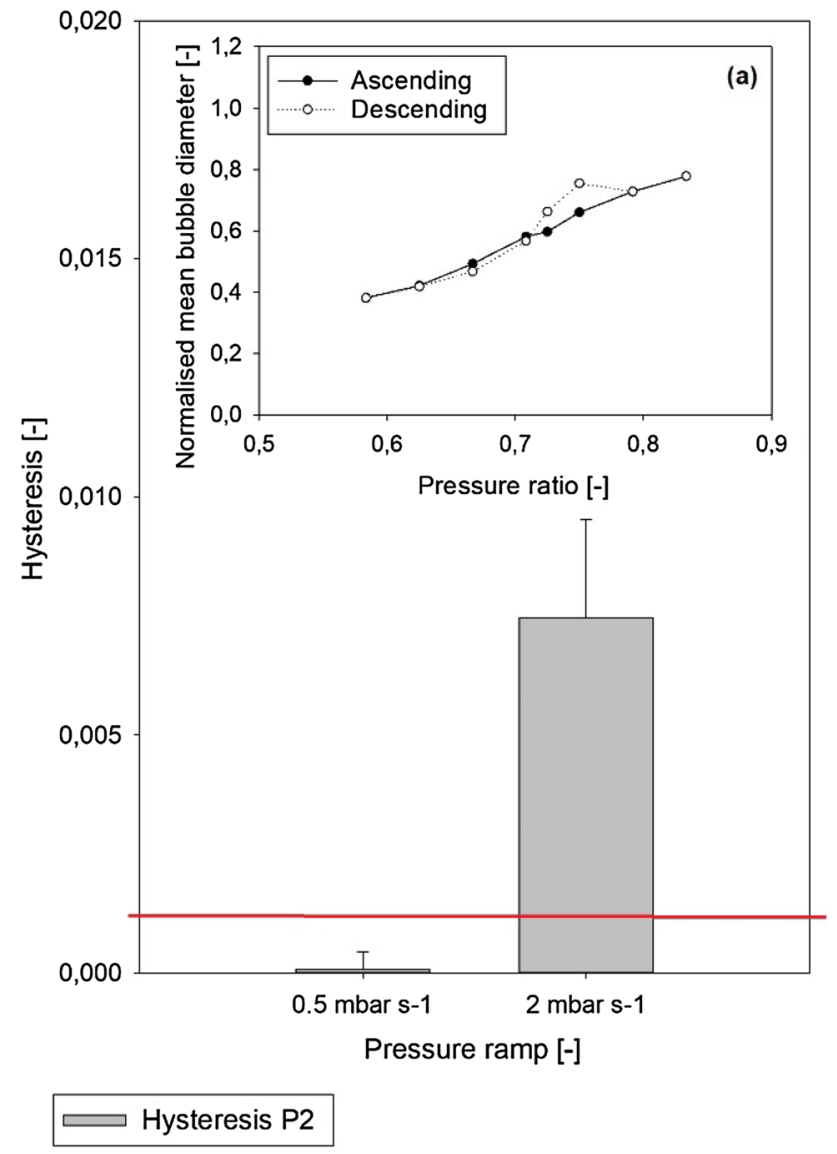

Fig. 7 Hysteresis evolution for $5 \mathrm{cmc}$ SDS $+40 \%$ (wt.) glycerol at high-pressure set ( $\mathrm{P} 2$, grey) for both pressure ramps (0.5 and $2 \mathrm{mbar} \mathrm{s}^{-1}$ ). In the inset, a typical curve for $\mathrm{P} 2$ at $2 \mathrm{mbar} \mathrm{s}^{-1}$

Finally, the hysteretic behaviour of the foam also depends on the level of deformation applied compared to the foam apparent yield stress for the specific device employed. The dependence upon the level of deformation is found only in the case of the $5 \mathrm{cmc}$ SDS + 40\% (wt.) glycerol (C). The rise in viscosity increases the foam yield stress, as more energy is required to make the foam flow at higher viscosity. Additionally, the yield stress for the other formulations presenting lower viscosities is considered as negligible in the range of pressures investigated.

\subsection{Effect of surface elasticity}

From past studies about foam flow in which drainage is occurring, it has been shown that the liquid velocity varies as a power law with the liquid flow rate (Pitois et al. 2005; Feitosa and Durian 2008; Stevenson and Li 2010). The power law exponent depends upon the mobility of the surface of the bubbles. If the interface is rigid, the liquid flow between the bubbles is Poiseuille-like whereas for mobile interface the flow is plug-like. 


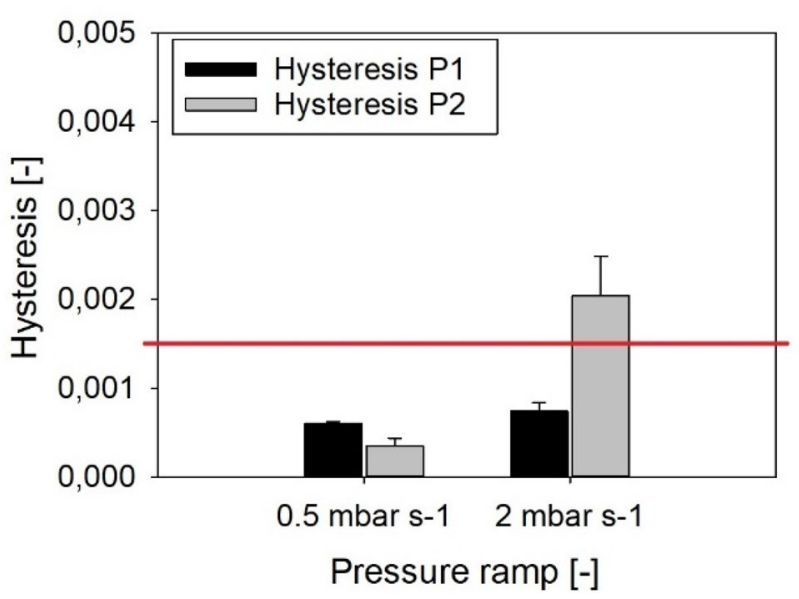

Fig. 8 Hysteresis evolution for $5 \mathrm{cmc}$ SDS $+0.15 \mathrm{~g} \mathrm{~L}^{-1} \mathrm{DOH}$ with the pressure ramp at low- (P1, black) and high- $(\mathrm{P} 2$, grey) pressure sets

$5 \mathrm{cmc}$ SDS
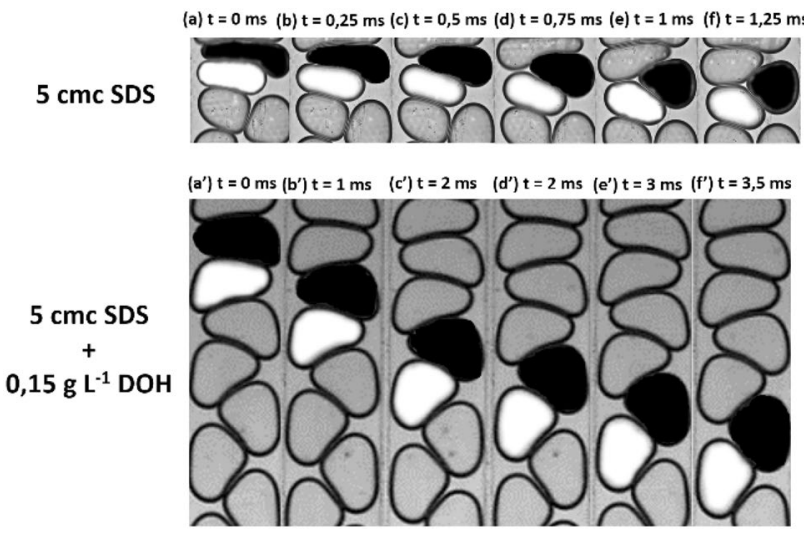

Fig. 9 Time lapses of the T1 bubble rearrangements for a fixed pressure set $\left(P_{\text {gas }}=400 \mathrm{mbar}, P_{\text {liq }}=600 \mathrm{mbar}\right)$ for $5 \mathrm{cmc}$ SDS from $(\mathrm{a}-\mathrm{f})$ versus $5 \mathrm{cmc} \operatorname{SDS}+0.15 \mathrm{~g} \mathrm{~L}^{-1}$ from $\left(\mathrm{a}^{\prime}-\mathrm{f}^{\prime}\right)$

The surface mobility depends primarily on the surfaceactive species: surfactants such as SDS will lead to mobile surfaces, whereas surfactant mixtures such as SDS-DOH mixture will create rigid surfaces. In fact, DOH molecules adsorb at the gas-liquid interface reducing the surfactant mobility in the plateau borders (Karakashev and Nguyen 2007). Indeed, when the surfaces of the plateau borders are said to be "rigid", the molecules' velocity at the interface tends towards zero (Dame et al. 2005). It has been also reported by Saint-Jalmes et al. (2004) that the surface dilatational viscosity increased from $0.08 \mu \mathrm{Pa} \mathrm{m} \mathrm{s}$ for SDS to $1.8 \mu \mathrm{Pa} \mathrm{m}$ s for SDS-DOH solutions for surfactant concentrations above the cmc.

The surface behaviour also relies on the foaming solution viscosity and on the bubble size. A transition between rigid and mobile surface behaviour can be noted while increasing bulk viscosity or bubble size (Safouane et al. 2006; Gauchet et al. 2015). Here, the continuous phase viscosity is steady as illustrated in Fig. 1.

From Fig. 8, both values for the hysteresis at 0.5 and $2 \mathrm{mbar} \mathrm{s}^{-1}$ at P1 (corresponding to 600 mbar liquid inlet pressure for a gas pressure ranging from 350 to $620 \mathrm{mbar}$ ) can be considered as negligible.

At P2 (corresponding to 800 mbar liquid inlet pressure for a gas pressure ranging from 550 to $820 \mathrm{mbar}$ ), the jump in ramp causes the hysteresis to rise above the reference value although marginally. Thus, depending on the kind of deformation at the wall applied, the increase in surface elasticity can lead to a rise in hysteresis.

From the existing relationship described by Durand and Stone (2006) limited to the case of dry foams at low strain rate regime, the $\mathrm{T} 1$ bubble rearrangement relaxation time $\tau_{T 1}$ can be expressed. The latter is described as the product of the shear rate times the ratio of surface dilatational viscosity $\kappa$ over the surface tension $\gamma$ such as $\tau_{\mathrm{T} 1} \approx \frac{\kappa}{\gamma} \times \dot{\gamma}$.

In this specific case, for a fixed surface tension, the duration of plastic events should increase due to the rise in the surface elasticity.

For the present case, the duration of a single $\mathrm{T} 1$ event is estimated for a fixed pressure set (400 and $600 \mathrm{mbar}$ gas and liquid pressures, respectively) by image analysis. Thus, the values 1.25 and $3.5 \mathrm{~ms}$ are obtained for the reference solution and for the solution containing $\mathrm{DOH}$, respectively, as represented in Fig. 9.

Above a critical level of deformation, foam undergoes a topological rearrangement between neighbouring bubbles. During this rearrangement, the foam stores elastic energy until it reaches saturation. This saturation is the reason of the existence of a yield stress (Marze et al. 2008).

In the present case, during the ascending ramp, the elastic energy storage increases with the ascending deformation until complete transition from two-row to bamboo pattern. Then, a steady plateau of high deformation at the wall is set for $60 \mathrm{~s}$ and then the descending ramp starts.

It is found that the hysteresis occurs either at the start of the high deformation plateau or following this step, at the start of the descending ramp. It is considered that the system cannot relax properly if it is submitted to an increasing deformation. The system is not able to relax this excess of elastic energy during the ascending ramp as the deformation increases.

The excess of elastic energy causes a local strain to build up. The accumulation of local strain triggers the hysteresis and the structural retardation observed in the channel. By extending the duration of the rearrangements, the amount of total elastic energy stored is heightened and so is the hysteresis. A retardation phenomenon is occurring due to the excess of elastic energy stored. By increasing the pressure set, the deformation at the wall is augmented. 
The bubble rearrangement relaxation time $\tau_{\mathrm{T} 1}$ is expressed as the product of the shear rate times the ratio of surface dilatational viscosity $\kappa$ over the surface tension $\gamma$. Thereby, by increasing the deformation at the wall and the shear rate applied, $\tau_{\mathrm{T} 1}$ increases linearly with the deformation. So, at higher deformation/pressure set, the amount of stored energy and hysteresis are enhanced. Similarly, the rise in pressure ramp (i.e., more energy per second to be stored) causes an increase in the hysteresis.

\section{Conclusions}

In this work, a novel approach to evaluate qualitatively foam hysteresis in a microchannel was established. The foam response to a gradual deformation at the wall was studied based on the transition from the two-row to the bamboo pattern. The impact of different parameters from the foaming liquid viscosity to the surface elasticity was studied on the foam recovery properties.

Throughout the different solutions investigated, the following observations were made:

1. A viscosity increase in the foaming solution for a constant surface tension leads to a retardation effect causing hysteresis both at low and high ranges of deformation.

2. An increase in the foaming liquid surface tension emphasizes the hysteresis and the resulting retardation effect depending on the level of deformation applied.

3. A higher surface elasticity creates a smaller but nonnegligible hysteresis due to an excess in elastic energy caused by the increase of the duration of the bubble rearrangements.

The approach introduced here has potential applications in a wide range of industrial processes where foams are generated and submitted to a series of deformation along the process line. This method could lead to the development of an accessible quality control tool to study the parameters (surfactant nature and properties, viscosity, viscoelasticity of the continuous phase, etc.) impacting significantly the foam recovery properties. Additionally, it could permit to study foam time dependency at micron scale, important property when microfluidics is used to generate or to study foams for industrial purposes from the generation of solid foams (Drenckhan and Langevin 2010; Andrieux et al. 2018) to the study of foam flow through porous materials such as in the enhanced oil recovery (EOR) application (Nguyen et al. 2014; Quennouz et al. 2014).

Acknowledgements The authors would like to thank Arnaud SaintJalmes for our fruitful discussions.
Open Access This article is distributed under the terms of the Creative Commons Attribution 4.0 International License (http://creativeco mmons.org/licenses/by/4.0/), which permits unrestricted use, distribution, and reproduction in any medium, provided you give appropriate credit to the original author(s) and the source, provide a link to the Creative Commons license, and indicate if changes were made.

\section{References}

Andrieux S, Drenckhan W, Stubenrauch C (2017) Highly ordered biobased scaffolds: from liquid to solid foams. Polymer. https:// doi.org/10.1016/j.polymer.2017.04.031

Andrieux S, Drenckhan W, Stubenrauch C (2018) Generation of solid foams with controlled polydispersity using microfluidics. Langmuir. https://doi.org/10.1021/acs.langmuir.7b03602

Asnacios RH, Cohen-Addad S, Asnacios A (1999) Rheological memory effect in aqueous foam. EPL (Europhys Lett) 48(1):93

Bauer WAC et al (2010) Hydrophilic PDMS microchannels for highthroughput formation of oil-in-water microdroplets and waterin-oil-in-water double emulsions. Lab Chip Miniat Chem Biol 10:1814-1819. https://doi.org/10.1039/c004046k

Bekkour K, Scrivener O (1998) Time-dependent and flow properties of foams. Mech Time-Depend Mater 2:171-193. https://doi. org/10.1023/a:1009841625668LB-Bekkour

Chung K et al (2009) Fabricating scaffolds by microfluidics. Biomicrofluidics 3:22403. https://doi.org/10.1063/1.3122665

Cohen-Addad S, Höhler R (2014) Rheology of foams and highly concentrated emulsions. Curr Opin Colloid Interface Sci 19(6):536548. https://doi.org/10.1016/j.cocis.2014.11.003

Costantini M et al (2015) Microfluidic foaming: a powerful tool for tailoring the morphological and permeability properties of sponge-like biopolymeric scaffolds. ACS Appl Mater Interfaces 7:23660-23671. https://doi.org/10.1021/acsami.5b08221

Cubaud T, Ulmanella U, Ho C-M (2006) Two-phase flow in microchannels with surface modifications. Fluid Dyn Res 38:772-786. https ://doi.org/10.1016/j.fluiddyn.2005.12.004

Dame C et al (2005) Relations between physicochemical properties and instability of decontamination foams. Colloids Surf, A 263:210 218. https://doi.org/10.1016/j.colsurfa.2004.12.053

de Souza Mendes PR (2009) Modeling the thixotropic behavior of structured fluids. J Nonnewton Fluid Mech 164:66-75. https:// doi.org/10.1016/j.jnnfm.2009.08.005

Drenckhan W, Langevin D (2010) Monodisperse foams in one to three dimensions. Curr Opin Colloid Interface Sci 15:341-358. https:// doi.org/10.1016/j.cocis.2010.06.002

Drenckhan W et al (2005) Rheology of ordered foams-on the way to discrete microfluidics. Coll Surf A Physicochem Eng Aspects 263:52-64. https://doi.org/10.1016/j.colsurfa.2005.01.005

Durand M, Stone HA (2006) Relaxation time of the topological T1 process in a two-dimensional foam. Phys Rev Lett 97:226101. https://doi.org/10.1103/PhysRevLett.97.226101

Feitosa K, Durian DJ (2008) Gas and liquid transport in steady-state aqueous foam. Eur Phys J E 26:309-316. https://doi.org/10.1140/ epje/i2007-10329-6LB-Feitosa

Galindo-Rosales FJ, Alves MA, Oliveira MSN (2013) Microdevices for extensional rheometry of low viscosity elastic liquids: a review. Microfluid Nanofluid 14:1-19. https://doi.org/10.1007/s 1040 4-012-1028-1LB-Galindo-Rosales2013

Garstecki P, Whitesides GM (2006) Flowing crystals: nonequilibrium structure of foam. Phys Rev Lett 97:24503. https://doi. org/10.1103/PhysRevLett.97.024503

Gauchet S, Durand M, Langevin D (2015) Foam drainage. Possible influence of a non-newtonian surface shear viscosity. 
J Coll Interface Sci 449:373-376. https://doi.org/10.1016/j. jcis.2014.12.060

Guillot P et al (2006) Viscosimeter on a microfluidic chip. Langmuir 22:6438-6445. https://doi.org/10.1021/la060131z

Guo F, Aryana S (2016) An experimental investigation of nanoparticlestabilized $\mathrm{CO}_{2}$ foam used in enhanced oil recovery. Fuel 186:430 442. https://doi.org/10.1016/j.fuel.2016.08.058

Höhler R, Cohen-Addad S (2005) Rheology of liquid foam. J Phys Condens Matter 17:R1041. http://stacks.iop.org/0953-8984/17/ $\mathrm{i}=41 / \mathrm{a}=\mathrm{R} 01$. Accessed Sept 2018

Karakashev SI, Nguyen AV (2007) Effect of sodium dodecyl sulphate and dodecanol mixtures on foam film drainage: examining influence of surface rheology and intermolecular forces. Colloids Surf A 293:229-240. https://doi.org/10.1016/j.colsurfa.2006.07.047

Kim P et al (2008) Soft lithography for microfluidics: a review. BIiochip J 2:1-11

Koser AE et al (2013) Measuring material relaxation and creep recovery in a microfluidic device. Lab Chip 13:1850-1853. https://doi. org/10.1039/c3lc41379a

Laporte $\mathrm{M}$ et al (2016) Characteristics of foams produced with viscous shear thinning fluids using microchannels at high throughput. J Food Eng 173:25-33. https://doi.org/10.1016/j.jfood eng.2015.10.032

Larson RG (2015) Constitutive equations for thixotropic fluids. J Rheol 59:595-611. https://doi.org/10.1122/1.4913584

Mark AE, Michael AJ, Bruce KG (2008) Determining the optimal PDMS-PDMS bonding technique for microfluidic devices. J Micromech Microeng 18:67001

Marze S, Langevin D, Saint-Jalmes A (2008) Aqueous foam slip and shear regimes determined by rheometry and multiple light scattering. J Rheol 52:1091. https://doi.org/10.1122/1.2952510

Marze S, Guillermic RM, Saint-Jalmes A (2009) Oscillatory rheology of aqueous foams: surfactant, liquid fraction, experimental protocol and aging effects. Soft Matter 5:1937-1946. https://doi. org/10.1039/B817543H

Mewis J (1979) Thixotropy-a general review. J Nonnewton Fluid Mech 6:1-20. https://doi.org/10.1016/0377-0257(79)87001-9

Mewis J, Wagner NJ (2009) Thixotropy. Adv Coll Interface Sci 147148:214-227. https://doi.org/10.1016/j.cis.2008.09.005

Mezger TG (2014) The rheology handbook, 4th Edition for users of rotational and oscillatory rheometers. 4th Edition. Edited by European Coating Tech Files. Vincents Network, Hanover

Miquelim JN, Da Silva Lannes SC (2009) Egg albumin and guar gum influence on foam thixotropy. J Texture Stud 40:623-636. https:// doi.org/10.1111/j.1745-4603.2009.00201.x
Nguyen P, Fadaei H, Sinton D (2014) Pore-scale assessment of nanoparticle-stabilized $\mathrm{CO}_{2}$ foam for enhanced oil recovery. Energy Fuels 28:6221-6227. https://doi.org/10.1021/ef5011995

Pipe CJ, McKinley GH (2009) Microfluidic rheometry. Mech Res Commun 36:110-120. https://doi.org/10.1016/j.mechrescom .2008 .08 .009

Pitois O, Fritz C, Vignes-Adler M (2005) Liquid drainage through aqueous foam: study of the flow on the bubble scale. J Colloid Interface Sci 282:458-465. https://doi.org/10.1016/j.jcis.2004.08.187

Quennouz N et al (2014) Microfluidic study of foams flow for enhanced oil recovery (EOR). Oil Gas Sci Technol 69:457-466. https://doi. org/10.2516/ogst/2014017

Raven JP, Marmottant P (2006) Periodic microfluidic bubbling oscillator: insight into the stability of two-phase microflows. Phys Rev Lett 97:154501. https://doi.org/10.1103/PhysRevLett.97.154501

Raven JP, Marmottant P (2009) Microfluidic crystals: dynamic interplay between rearrangement waves and flow. Phys Rev Lett 102:84501. https://doi.org/10.1103/PhysRevLett.102.084501

Ruiz CC, Díaz-López L, Aguiar J (2008) Micellization of sodium dodecyl sulfate in glycerol aqueous mixtures. J Dispers Sci Technol 29:266-273. https://doi.org/10.1080/01932690701707571

Safouane $\mathrm{M}$ et al (2006) Viscosity effects in foam drainage: newtonian and non-newtonian foaming fluids. Eur Phys J E 19:195-202. https://doi.org/10.1140/epje/e2006-00025-4

Saint-Jalmes A (2006) Physical chemistry in foam drainage and coarsening. Soft Matter 2:836-849. https://doi.org/10.1039/B606780H

Saint-Jalmes A, Zhang Y, Langevin D (2004) Quantitative description of foam drainage: transitions with surface mobility. Eur Phys J E 15:53-60. https://doi.org/10.1140/epje/i2004-10036-xLB-Saint -Jalmes 2004

Schneider CA, Rasband WS, Eliceiri KW (2012) NIH Image to ImageJ: 25 years of image analysis. Nat Methods 9:671-675

Skurtys O, Bouchon P, Aguilera JM (2008) Formation of bubbles and foams in gelatine solutions within a vertical glass tube. Food Hydrocoll 22:706-714. https://doi.org/10.1016/j.foodh yd.2007.02.003

Stevenson P, Li X (2010) A viscous-inertial model of foam drainage. Chem Eng Res Des 88:928-935. https://doi.org/10.1016/j.cherd 2010.01.014

Yekeen N et al (2018) A comprehensive review of experimental studies of nanoparticles-stabilized foam for enhanced oil recovery. J Petrol Sci Eng 164:43-74. https://doi.org/10.1016/j.petrol.2018.01.035

Publisher's Note Springer Nature remains neutral with regard to jurisdictional claims in published maps and institutional affiliations.

\section{Affiliations}

\section{Leslie Labarre $^{1} \cdot$ Daniele Vigolo $^{1}[$}

Daniele Vigolo

d.vigolo@bham.ac.uk
School of Chemical Engineering, University of Birmingham, Birmingham B15 2TT, UK 